\title{
Las astucias de la razón neoliberal. Los usos del rechazo en la reproducción del Modelo Neoliberal en Chile (1990-2010s)
}

\author{
Juan Jiménez A. ${ }^{1}$ Universidad Tecnológica Metropolitana, Chile
}

17 de diciembre de 2020

\begin{abstract}
Resumen
La relación subjetiva de la población chilena con el modelo económico neoliberal ha sido ampliamente discutida. Para comprender estas relaciones se ha usado tradicionalmente el concepto de legitimación. En años recientes se ha planteado al neoliberalismo como un sistema que no opera a través de legitimación la. Usando el caso de Chile, argumentaremos que la subjetividad se incorpora a la reproducción por un camino más complejo. Así, la subjetividad chilena sobre el neoliberalismo está compuesta por cuatro capas: una evaluación positiva del estándar de vida, una naturalización del modelo, un mandato del esfuerzo y una crítica moral. En todas estas capas el sujeto, incluso si se acerca, muestra una distancia con el modelo. Sin embargo, el rechazo que está detrás de esa distancias genera las conductas que reproducen el modelo: El neoliberalismo usó el descontento para reproducirse.

\section{Palabras clave}

Neoliberalismo, subjetividad, Chile
\end{abstract}

\begin{abstract}
The subjective relationship of the Chilean population with the neoliberal economic model has been widely discussed. The concept of legitimacy was the traditional frame to understand these relationships, under the idea that no illegitimate system can subsist. In recent years neoliberalism has been presented as a system that does not require subjective legitimation. Using the case of Chile, we will argue that subjectivity matters for reproduction through a more complex path. On the one hand, Chilean subjectivity is formed through four

\footnotetext{
${ }^{1}$ E-mail: j.jimeneza@utem.cl Una primera versión de este texto fue presentado en el III Congreso Latinoamericano de Teoría Social, Buenos Aires, 31 de Julio al 2 de agosto de 2019. Mesa 31. Teorías críticas del neoliberalismo. Se agradecen comentarios de Rodrigo Márquez, Felipe Torralbo, Miguel Urrutia y Andrea Gartenlaub.
} 
different layers: a positive evaluation of the standard of living, a naturalization of the model, a mandate for effort, and a moral critique. In all these layers what it appears to be a neoliberal subjectivity shows a distance with the model. On the other hand, the rejection behind these distances generates behaviours that reproduce it: Neoliberalism used discontent to reproduce itself. The refuge in personal life created the consumer and the worker that the model sought.

\section{Keywords}

Neoliberalism, subjectivity, Chile

\section{Introducción. La discusión del neoliberalismo como concepto}

El neoliberalismo, como casi todo concepto muy usado, se dice de múltiples formas. Aquí lo definiremos como un concepto histórico referido a un proceso que ocurre en un momento determinado: las prácticas que emergen en las últimas décadas del siglo XX en reacción contra la 'modernidad organizada' (Wagner 1997): Menor presencia del Estado y aumento de la importancia del mercado, desregulación y globalización económica, fuerte presencia de capitalismo financiero, todo lo cual ocurre luego tras un momento con mayor presencia estatal (Gray 1998, Osorio 2004, Clarke, 2005, Dunn 2016). Esto no implica un estado débil: se ha requerido de un Estado fuerte para implementar los mercados en formas neoliberales (lo cual tiene precedentes: cuando el Estado se retiró de la economía del Reino Unido en la revolución industrial era uno de los estados más fuertes de Europa, Mokyr, 2009). Podemos llamar a esto neoliberalismo incluso si se acepta que no hay nada nuevo en ello (como argumenta Dunn 2016); sigue siendo útil como un 'broad indicator of the historical turn in macropolitical economy’ (Venugopal 2015: 182).

Además de dichas prácticas el neoliberalismo es el conjunto de ideas que las justifican y promueven; y nuevamente lo central es el hecho que ellas se desarrollan después de un período de dominancia de ideas contrarias: El neoliberalismo es aquella posición que defiende los procesos de liberalización. Se radicaliza la perspectiva que la regulación estatal es ineficiente o inmoral y se enfatiza que el despliegue del mercado equivale al despliegue de la libertad. Una sociedad con un proyecto colectivo o con auto-determinación colectiva es una forma de tiranía, que limita la única libertad válida: la que ocurre al interior del mercado (Slobodian 2018, Milanovic, 2019). 
El neoliberalismo también ha sido analizado como posición subjetiva, conformando una manera de ser. Si la condición neoliberal es una de creciente relevancia del mercado, la subjetividad neoliberal sería aquella donde las personas se piensan a sí mismas como actores mercantiles. Discusiones sobre un self emprendedor (Bröckling, 2015; Roper, Ganesh, y Inkson, 2011), o como al exacerbar la competencia se construye un individuo que se piensa a sí mismo como empresa (Laval y Dardot, 2013) de alguien que se 'vende a sí mismo' en todo momento corresponden a esta mirada ${ }^{3}$. Un punto central en esta discusión es diferenciar esa construcción subjetiva de una toma de posición ideológica. Alguien puede, en la práctica, asumir una visión de sí centrada en la competencia y en la venta de sí, sin necesidad de asumir una visión neoliberal sobre la sociedad, el papel del mercado o la imagen del Estado.

\section{El Neoliberalismo y la relación entre ideología y subjetividad}

La pregunta por la relación entre subjetividades y las condiciones y estructuras sociales es tradicional en sociología. Resultaba común afirmar que la pura operación de una estructura no permite su reproducción; se requiere además una operación subjetiva. La subjetividad y la estructura deben estar en tándem para que la sociedad opere (así Dubet, 1994 en relación con la tradición sociológica, o Morandé, 2017, sobre el pensamiento ilustrado en general). Todo sistema requeriría de apoyo a través de creencias que lo postulen como "bueno" o al menos como "natural" (por ejemplo Bourdieu 2015); requiere entonces sustento ideológico, legitimidad. Las alternativas a la ideología -la violencia o el contrato- (Lichbach y Seligman, 2000) no eran suficientes: el 'cemento de la sociedad' requiere también normas (Elster 1989). La cohesión social se genera en la dinámica entre la legitimidad -normas- y los procesos factuales -la coerción o el efecto instrumental de los contratos- (Habermas, 1998). En cualquier caso, en la tradición sociológica la subjetividad opera y se vuelve relevante a través de la legitimidad.

\footnotetext{
${ }^{3}$ Esto sería (si esas posiciones son correctas) una diferencia con el liberalismo clásico que se constituía en la diferencia entre el espacio doméstico, donde no se vive bajo el mercado, y el espacio 'público' donde eso ocurre (por ejemplo, Hobsbawm, 1975 sobre el mundo burgués en la era de su total hegemonía). Bajo el neoliberalismo la orientación de mercado sería omnipresente.
} 
Con el neoliberalismo aparece una pregunta. Existe la intuición que la subjetividad en el neoliberalismo no opera a través de la ideología (y más en general, que los procesos simbólicos no pueden analizarse sólo con esos lentes, Reygadas 2015: 43). El concepto de gubernamentalidad de Foucault $(2000,2007)$, una intervención que no se basa en la operación de una lógica de soberanía o de manejo de prácticas disciplinarias, sino que controla a las personas usando sus rasgos para lograr que ellas hagan lo que tienen que hacer (Agamben, en El Reino y la Gloria, 2016: 461-534, ha analizado las raíces profundas de ello en la teología cristiana), ha resultado importante aquí. La idea de Deleuze (1991) de sociedades de control sigue una línea similar: mecanismos que logran que los sujetos operen con acuerdo a los requerimientos estructurales sin usar ideología. Durand (2004: 59-62) enfatiza cómo el puro ritmo y características de los procesos de trabajo generan conformidad. Lordon (2013: 94) ha discutido como a través de las emociones el neoliberalismo genera afectos positivos directamente en el trabajo. El ejemplo que da Bourdieu al inicio de Las Estructuras Sociales de la Economía (2000), ilustra estas dinámicas subjetivas que no pasan por legitimación ideológica: un grupo de niños ingleses crean un fondo de compensación por castigos paternales y tienen que incluir en sus 'leyes' el que no serán compensados por comportamientos que buscaban ex profeso ser castigados. La racionalidad económica era algo que tenían incorporado en su habitus.

Esta idea de un control que es tan intrínseco al operar que ya no requiere de un mecanismo ideológico ulterior no es completamente ajena a la tradición. En las páginas finales de la Ética Protestante de Weber se puede encontrar un eco de ello ('la jaula de hierro' en la frase de la traducción de Parsons que ha hecho historia). Sin embargo, el ejemplo nos muestra que un sistema que no requiere de un soporte ideológico aparecía en la tradición bajo la forma de un monstruo.

Esta intuición sobre el neoliberalismo, empero, mantiene elementos básicos sobre la relación entre subjetividad y estructura. En el argumento tradicional subjetividad y reproducción social se relacionan de manera directa: Cuando la posición subjetiva es positiva hacia la estructura, reproduce; lo contrario cuando es negativa. Incluso cuando se abandonó el funcionalismo se mantuvo la idea. El concepto de habitus en Bourdieu (1980, 2015) sigue el mismo camino de una dinámica subjetiva que se imbrica en la reproducción social por consonancia. La reproducción social necesita que el agente se someta a sus 
instituciones, y cuando ellos observan alternativas a éstas la reproducción social pierde fluidez (Boltanski 2009: 93-98).

Esto no cambia al abandonar la idea de legitimidad. Así, Lordon (2013) encuentra vacío dicho concepto (no habría diferencia entre una institución legítima y una existente), reemplazándolo por una dinámica de poder y emociones: En la reproducción esas emociones son positivas hacia la estructura, y causan crisis cuando son negativas. Más en general, el sujeto neoliberal sería uno que reproduciría el neoliberalismo no por su acuerdo ideológico con éste, sino que porque en su actuar (no en su creencia) se pliega a las exigencias del neoliberalismo. Una acción neoliberal implicaría una subjetividad neoliberal donde 'capitalism has successfully transformed humans into calculating machines endowed with limitless needs' (Milanovic, 2019: 196). La diferencia entre la idea tradicional y la perspectiva de una subjetividad neoliberal ocurre en el mecanismo que une subjetividad y reproducción (ya sea con legitimación, ya sea conformando disposiciones a actuar), pero en ambos casos la subjetividad opera a través de una dinámica de aceptación/rechazo para la reproducción social.

En general, la subjetividad se conceptualiza con el prisma de la estructura. Cuando la acción reproduce la estructura, 'nada' sucede en ella; la acción propiamente acción es cuando los agentes cambian el sistema (así en Dubet 1994: 127, Touraine 2013 o White 2008). No hay eventos en la reproducción, ella consistiría meramente en que las instituciones son exitosas generando las creencias, emociones y posiciones subjetivas 'correctas' (una crítica en Moore 2011). Si, por ejemplo, el habitus es una forma de reproducción, entonces se sigue que Bourdieu no tendría una noción fuerte de agencia (King 2000, Martuccelli 2013), y entonces se necesita ir más allá de Bourdieu (Atkinson 2010): si los agentes sociales reproducen el sistema, entonces nada relevante ocurre en la agencia. Lo que está detrás de estas ideas es la noción que lo único que requiere una estructura para reproducirse es lograr que las personas la acepten y que una estructura completamente legítima no tiene fuerzas internas para cambiar. Así, luego de largas discusiones, la teoría social vuelve a ser parsoniana, y mantiene el mismo enlace entre subjetividad y reproducción social que el funcionalismo clásico.

Aquí argumentaremos contra esta común posición: Que la relación entre subjetividad y reproducción no es tan directa y que existe un papel para la subjetividad en las dinámicas de 
la reproducción más compleja que una de aceptar=reproducción (y rechazar=sin reproducción). El ejemplo de la cristiandad temprana ilustra a que nos referimos con ello: El cristiano pagaba impuestos y obedecía al César, pero su subjetividad (su imagen de sí) no estaba radicada en este mundo, y su subjetividad se basaba en esa distinción: ser un cristiano es estar en el mundo sin ser $d e l$ mundo. Esa subjetividad era ajena a esa estructura, pero fue -al menos durante un tiempo importante- compatible con ella.

Sólo separando con claridad la subjetividad de los procesos de reproducción es posible que un aserto como 'el neoliberalismo genera una subjetividad que no opera por legitimidad' sea relevante. Si la subjetividad neoliberal es equivalente al hecho que las personas se comportan de acuerdo a las exigencia del sistema, entonces siempre que el sistema se reproduce existiría una subjetividad neoliberal (y se repite este concepto vacío como Lordon calificaba a la legitimidad). Sólo si existe la posibilidad que una práctica existente no genere de manera automática una subjetividad acorde con ella, no es tautológico decir que una práctica genera una subjetividad. Y a su vez, para que la subjetividad tenga importancia en la descripción de la operación de una estructura se requiere que sus dinámicas efectivamente tengan consecuencias para ella (de otra forma ni siquiera es 'superestructura'). La combinación de ambas ideas genera el espacio de una subjetividad que puede ser parte de dinámicas de reproducción sin ser mero plegamiento a la estructura.

En las siguientes secciones de este texto intentaremos mostrar que para comprender la relación subjetiva de la población chilena con el neoliberalismo es en esos espacios que se requiere pensar. A continuación, describiremos en líneas generales la implementación del neoliberalismo y la discusión sobre la subjetividad en Chile; y en la sección subsiguiente describiremos en mayor detalle la relación de la subjetividad con el neoliberalismo, y volveremos después a las consecuencias de ello sobre esta discusión más general sobre la subjetividad.

\section{Chile como sociedad de 'neoliberalismo integral'}

Chile es uno de los casos más tempranos y más sistemáticos de implantación del neoliberalismo, dirigido por un grupo que no solamente intentaba construir una economía de mercado, sino una "sociedad de mercado" (Gárate 2012, Clark 2017, Medina-Zárate y Uchôa de Oliveira 2019): el mercado organizando la 'vida productiva' y también la 
'reproducción de la vida', insertado de manera central en la educación o la salud (Canales 2019). Incluso si el neoliberalismo no es necesariamente una hegemonía coherente (Venugopal 2015, Welsh 2019: 16-19), Chile representa un caso donde el movimiento hacia el mercado como un principio organizador de la vida social se desarrolló de forma más integral y, entonces, más cercano a la hegemonía.

La dictadura militar instaura a finales de los '70 las reformas estructurales que imponen el modelo neoliberal (Gárate 2012: 256-283): Liberalización de la economía (desde aranceles hasta mercado laboral), privatización o limitación del Estado en los servicios sociales (salud, pensiones, educación); es el modelo que rigió los siguientes 40 años en Chile. Las modificaciones subsiguientes no superan lo que Garretón (2012) denominó 'neoliberalismo corregido'; los analistas que plantean la generación de un modelo distinto (Castells 2005, Calderón y Castells 2019: 32) no toman en cuenta esta continuidad de reformas básicas. En lo relativo al nivel ideológico, también en Chile la libertad se planteó como equivalente a un mercado ilimitado y los promotores del modelo defendieron un rechazo absoluto de la política (Brunner, 1981, Lechner, 2007, II: 137-180, publicado en 1982, Gárate, 2012), buscando una sociedad ordenada por el automatismo del mercado (Brunner 1983: 87-90).

Siendo Chile una sociedad donde el régimen neoliberal ha durado más tiempo y de forma más integral, se convierte en caso interesante para observar la subjetividad bajo el neoliberalismo. Un hito importante en este debate se abrió hacia finales de la década de los '90 cuando aparecieron dos textos críticos sobre un modelo que hasta ese momento se mostraba exitoso: Las paradojas de la modernización (PNUD Chile, 1998) y Chile actual: Anatomía de un mito (Moulian 1997).

El primero de ellos planteó lo que se conoció como la tesis del malestar, de un rechazo subjetivo amplio al modelo. Esta idea marcó de manera importante el debate posterior que prosiguió en buena parte con esa misma pregunta: ¿se acepta o no el modelo? Así, está la posición de Mayol de un movimiento desde una progresiva implantación de ideas favorables al modelo (Mayol et al 2013) a un crecimiento posterior de su crítica (Mayol 2012). También encontramos quienes postularon la existencia de una amplia satisfacción, y donde toda crítica era ya una que al interior del modelo o una que criticaba elementos que no son parte de éste (CEP, 2016; Oppliger y Guzmán, 2012). 
El texto de Moulian abrió otro espacio de discusión centrado en el posicionamiento de la persona: una concepción de sí centrada en ser consumidor en oposición a una identidad de ciudadano; y es en las prácticas generadas por el consumo 'libre', en el encadenamiento de la deuda, que el sistema alinea a la persona. Esta posición ha sido compartida tanto por analistas críticos del modelo, como el mismo Moulian (también Fagioli 2018 pensando allí en subjetividad emprendedora) como por quienes más bien lo aceptan (Tironi 2000, Navia 2008: 288-289). El neoliberalismo generaría un sujeto que se piensa a sí mismo en términos de elecciones y de cálculos (Canales 2019). Sin embargo, la evidencia que se aduce para esta tesis no suele pasar del que las personas efectivamente realizan comportamientos 'neoliberales', y a partir de ello se deduce una subjetividad, lo que no es suficiente.

La pregunta por las disposiciones subjetivas sólo se despliega cuando se abandona esa presunción que la conducta es suficiente para describirla. Un ejemplo de ello son estudios en instituciones educacionales que muestran como el uso de pruebas estandarizadas genera efectos subjetivos: al orientar la acción de los agentes se exacerba una lógica de comparación y competencia (Falabella 2019: 17).

La investigación cualitativa de Araujo y Martucelli (2012) en Desafíos Comunes es una de las más interesantes al respecto mostrando los procesos subjetivos a través de los cuales las personas responden a las exigencias en el trabajo o a su familia. Esa dimensión subjetiva está separada de la adhesión ideológica, y la respuesta esencial es que si bien el modelo produce dinámicas subjetivas en respuesta a sus exigencias (también Araujo, 2014); lo hace menos en la segunda dimensión: No serían personas que creerían ideológicamente en lo que dice el neoliberalismo. Pero incluso más allá de ello, ahí se observa en concreto la distancia entre la acción conforme y la subjetividad: No porque las personas, por ejemplo, sepan orientarse en una lógica de comparación y competencia se sigue que sean 'neoliberales': la disposición a seguir las exigencias del trabajo se sostiene a partir de una visión de la familia que no es neoliberal. A su vez, Mayol et al (2013: 162-3) muestran que la valoración del emprendimiento puede basarse en una búsqueda de la tranquilidad, lo cual hace dudar de si se está ante una lógica emprendedora. 


\section{Las formas de la relación entre subjetividad y neoliberalismo.}

En esta sección presentamos una descripción más detallada de las diversas maneras en que la subjetividad se relaciona con el neoliberalismo. Para comprender esta relación es necesario, al menos, distinguir cuatro capas. Primero, la evaluación del estándar de vida. Segundo, una visión cognitiva sobre cómo opera el mundo social. Tercero, una imagen de sí mismo. Cuarto, una evaluación ética del mundo social.

Es importante enfatizar que estas son formas no variantes de la relación. Cada persona incluye estas diferentes capas en su subjetividad. No deben ser pensadas como alternativas (que las personas están en la variante de la capa primera o de la cuarta capa). A su vez, al interior de cada capa hay distintas posturas, aunque en nuestra descripción apuntaremos al juicio más general. De esta forma se muestra la complejidad de los elementos que conforman la relación subjetiva con el neoliberalismo.

\section{a. La visión del estándar de vida}

Diversas encuestas muestran que la población tiene una imagen positiva de la historia de su estándar de vida (tabla 1). Si se les pregunta si viven o están en una situación mejor que sus padres a su misma edad, la mayoría responde de manera afirmativa; y esta percepción se fortaleció en el tiempo (subiendo entre 15 y 20 puntos entre 2007 y 2018, PUC 2018)

Tabla 1. Haciendo una comparación entre su vida y la que sus padres tenían a su edad:

\begin{tabular}{ccccc}
\hline & $\begin{array}{c}\text { Mucho mejor+ } \\
\text { Mejor }\end{array}$ & Igual & $\begin{array}{c}\text { Mucho } \\
\text { peor+ Peor }\end{array}$ & NS/NR \\
\hline Nivel de ingreso suyo & 67 & 21 & 9 & 3 \\
\hline La casa que ud. tiene & 65 & 23 & 7 & 5 \\
\hline Su trabajo le parece & 61 & 19 & 6 & 12 \\
\hline Su vida familiar es hoy día & 68 & 25 & 6 & 1 \\
\hline Su tiempo libre es & 66 & 21 & 12 & 1
\end{tabular}

Fuente: Encuesta Bicentenario 2018. 
Los chilenos perciben que sus niveles de consumo son mayores y que pueden acceder a bienes y servicios a los que no podían previamente. Los niveles de crecimiento de las últimas décadas y el salto en el ingreso per cápita son datos objetivos (Bolt et al 2018) que la subjetividad refrenda.

Ahor bien, esta percepción no se traduce automáticamente en una visión positiva del modelo. Se detecta, por ejemplo, una fuerte diferencia entre percepciones sobre la propia vida y sobre la sociedad. La encuesta CEP mayo 2019 muestra un promedio de satisfacción con la propia vida de 7,1 mientras que estiman sólo en un 5,3 la satisfacción de otras personas (CEP 2019). En estudios cualitativos la diferencia es aún más notoria: mientras el país se percibe de forma negativa, la conversación sobre la propia vida aparece más positiva. Las dos secuencias de conversaciones grupales que presentamos a continuación de un estudio del PNUD Chile muestra este contraste entre una mirada negativa sobre la sociedad (la primera) con la mirada sobre la propia vida, no exenta de problemas pero mejor (la segunda)

- ¿Se pueden decir garabatos?

$-($ Risas $)$

-Como las pelotas en realidad.

-Sí, muy mal, laboralmente...

-Es lo más suave que puedo decir

-Yo encuentro que bien, en este minuto económicamente estoy bien, estoy feliz con lo que tengo, bien.

-Yo no.

-Bien en el sentido en la parte económica, en la parte trabajo, en la parte salud, en eso estoy bien.

$$
\left(\text { PNUD 2016) }{ }^{5}\right.
$$

\footnotetext{
${ }^{5}$ Este estudio del PNUD se basó en 7 entrevistas grupales realizadas en Santiago durante el año 2015 (un grupo adulto NSE alto, un grupo adulto medio, un grupo adulto medio-bajo, un grupo adulto bajo, un grupo joven medio y alto, un grupo joven medio-bajo, un grupo dueñas de casa medio-bajo). En este texto usaremos citas tanto de la publicación como de las transcripciones de las entrevistas. Estas últimas serán referidas como PNUD Inv. progreso 2015.
} 
La diferencia ha generado debates sobre sus causas e interpretación (Beyer, 2015; Márquez, 2015; PNUD 2015), pero independiente de ello la diferencia como tal es reconocida. En algún sentido estamos ante una simple constatación de una experiencia ya vivida: Que el nivel de vida es más alto que en décadas anteriores. Una constatación sobre el pasado de la cual no se extraen más conclusiones.

La percepción de un aumento en el nivel de vida permite entender otros resultados comunes sobre Chile y que han sido usados para defender que la población aprueba el modelo: la extendida percepción de pertenecer a la clase media (Barozet y Fierro 2014: 152). Nuestro argumento es que las personas declaran ser clase media no tanto como una afirmación sincrónica sobre donde están posicionados en la estructura social, sino más bien como una afirmación diacrónica sobre su avance en la vida. El mejor estándar de vida implica que muchos no quieran identificarse como de 'clase baja' puesto que eso implicaría negar que viven en mejores condiciones. Dadas las alternativas que se les presentan en las encuestas, la alternativa más plausible es responder 'clase media'. Esta interpretación da cuenta del hecho que cuando las encuestas presentan una alternativa como 'clase trabajadora', que les permite reconocer su mejora en el estándar de vida sin decir que son clase media, ella se transforma en una respuesta común : Datos del 2019 muestran un $41 \%$ de identificación con 'clase trabajadora' comparable al $44 \%$ sumando las tres versiones de clase media que ofrece la encuesta (CEP 2019); y datos del 2009 también mostraban una menor percepción de clase media cuando aparece la posible respuesta 'clase trabajadora' (Jorrat 2014: 70). Un estudio usando viñetas (no categorías pre-establecidas) para crear autocategorizaciones muestra que un $68 \%$ se auto-identifica con la categorización inferior (MacClure et al 2019: 15). Cuando las personas dicen 'soy de clase media' lo que realmente plantean es 'mi vida ha mejorado'. La auto-identificación con la clase media no es tanto un voto de confianza en el neoliberalismo como la forma en que las personas reconocen su mayor estándar de vida bajo las alternativas usuales de respuesta en encuestas.

\section{b. La visión de mundo: El mundo opera con acuerdo al modelo neoliberal.}

El segundo juicio es sobre una pregunta más general sobre el mundo: ¿Cómo funciona y qué es posible hacer en él? 
El modelo 'mental' sobre cómo funciona el mundo es el de uno marcado por la competencia y por el mercado, donde todos se mueven en la búsqueda permanente de ganancia. Eso es lo 'normal' y lo que se aleja de éste es aberración o imposible (PNUD Chile 1998, 2002). Se hace difícil pensar la vida fuera del él, lo que no se limita a Chile. La idea que 'there is no alternative' (TINA) es de Margaret Thatcher y que es más fácil imaginarse el fin del mundo que el fin del capitalismo se ha repetido varias veces (por ejemplo, Žižek 2008: 7-42). El hecho que en la práctica el capitalismo sea el único sistema económico existente (Milanovic 2019), aunque con una serie de variantes, no todas 'neoliberales', también ayuda a producir la imagen de un solo mundo posible.

Ahora bien, esta falta de alternativas es una falta de alternativas exitosas. Toda discusión sobre posibles alternativas enfrenta el muro de 'las alternativas fracasaron'. La caída de los socialismos reales a principios de los '90 operó como inmunizador por décadas: cada vez que se pensaba una alternativa como posible reaparecía esta idea de fracaso (Appel y Orenstein 2016, Bockman 2019).

Esta mirada (que la sociedad de mercado es la orientación natural de la vida) es un aspecto donde coinciden la subjetividad de las personas y la ideología neoliberal. En la visión de los impulsores del modelo en Chile el orden del mercado era el orden natural de las cosas (Gárate 2012, Brunner 1983).

Sin embargo, sigue sin ser una capa plenamente equivalente a la ideología neoliberal. Para las personas este orden lo es no sólo porque es 'natural' sino además, y quizás aún más, porque grupos específicos así lo establecen. Habría aquí un problema del poder: algunos segmentos con mucho poder activamente limitan cualquier posibilidad de cambio. Existe una máquina muy poderosa frente a la cual nada se puede hacer (PNUD 2004) y 'los poderosos' siempre ganan

Entonces, en definitiva, ese poder es muy fuerte y tratar de..., de vulnerar y darlo vuelta ese poder es muy difícil, digamos. (NSE alto) Debe partir por parte de los empresarios, que son los sostenedores de este país, partiendo de ahí yo creo que ahí le darían autorización al Gobierno para poder hacer algún cambio. (NSE bajo) (PNUD 2015: 
Esta naturalización, además, no opera necesariamente, en distinción con la ideología, de manera positiva. Ese orden "natural" no es por ello un orden bueno, deseable. Si en el discurso neoliberal esa equivalencia es central y garantiza que se puede derivar la bondad del orden del hecho que sea natural, ello no ocurre en la subjetividad. Para usar una frase común en las conversaciones en Chile: 'es lo que hay', y la frase indica un orden negativo que no se puede modificar.

La forma en que el neoliberalismo se asentó en Chile puede explicar, quizás, estos resultados. Lo que éste hizo con éxito fue desmontar las alternativas, hacerlas 'increíbles', pero no construyó su propia justificación. El neoliberalismo no necesitó convencer de su propia bondad, sólo mostró el fracaso de otras alternativas. Por ello, se puede plantear que el mundo creado por el neoliberalismo es leído bajo otros parámetros e ideas. Incluso cuando alguien se mueve 'neoliberalmente' no lo hace leyendo la realidad como lo hace un neoliberal; y ese mundo es pensado desde fuera, bajo una lógica distinta.

Lo que las personas observan en el mundo neoliberal de la competencia y el mercado no fue un mundo de intercambios libres entre agentes a su vez libres (Gárate 2012), sino una máquina impersonal que coacciona a las personas y les niega su libertad (PNUD Chile 2002): un mundo despiadado donde 'el pez grande se come el chico', marcado por un individualismo que sería un protegerse sólo a sí mismo y una compulsión para consumir puesto que es ahí donde se juega el éxito (Aravena y Baeza 2015: 155). Este mundo neoliberal naturalizado no es leído neoliberalmente.

\section{c. La construcción de sujeto. El mandato del esfuerzo.}

La tercera capa refiere a la auto-identidad. Quizás es aquí donde resulta más necesario evitar malentendidos. Un ejemplo claro de ello son las discusiones sobre un self empredendor. Así, el trabajo por cuenta propia no es necesariamente "emprendimiento", incluso entre quienes prefieren este tipo de trabajo el motivo fundamental es el rechazo a la subordinación a un empleador más que un deseo de emprender (Acuña y Pérez 2005, Jiménez 2016), y la presencia de una fuerte proporción de empleo por cuenta propia (con actitudes positivas hacia éste y contra el asalariado) es parte del Chile previo al neoliberalismo y se observa ya en el siglo XIX (Salazar 2000, 2009, Undurraga 212). 
La expansión de ideas de esfuerzo y de mérito en la construcción de la auto-identidad (PNUD, 2016, Mayol et al 2013) es crucial. Y el hecho que ello no debe identificarse sin más como neoliberal se muestra en que ellas se enraízan en un sentido muy profundo de deber hacia sus familias (Castillo 2016, Araujo y Martuccelli 2012). Las conversaciones grupales del estudio PNUD Inv. progreso 2015 muestran un fuerte despliegue de la idea de esfuerzo, permitiendo plantear la existencia de un mandato del esfuerzo. Cuando las personas en las conversaciones grupales muestran que no se orientan por dicha idea, reciben una fuerte crítica al interior de los grupos. Las personas se auto-exigen que 'no digas que no puedes'. Existe una auto-afirmación alrededor de este mandato: cuando lo acatan las personas se perciben de forma positiva y dignas de admiración. La importancia del esfuerzo aparece también al observar narraciones de historias laborales, donde incluso cuando menos se destaca la autonomía del sujeto, el esfuerzo y el sacrificio operan como ejes que dan sentido a la trayectoria individual (Gaete y Soto 2012: 57)

Yo trabajo todos los días, encuentro que va en uno, también como dicen acá los compañeros, dicen que va en la persona en querer esforzarse, salir adelante y buscar no más, si a uno le gusta vivir bien, cómodo, tienes que esforzarte, trabajar y superarte cada día no más (NSE Bajo) No digas que no puedes. (Mujeres dueñas de casa, NSE Medio-Bajo) (PNUD 2016)

Por otro lado, las mismas personas establecen que no todos siguen esa orientación a través de un doble límite: Hacia abajo (los que no se esfuerzan, y aquí se distingue entre pobres 'decentes', trabajadores, y aquellos que no lo serían, ver Martínez y Palacios, 1996) y hacia arriba (los ricos que no necesitan esforzarse). Es importante enfatizar este último límite porque puede radicalizarse en el juicio que las dificultades para surgir son producto de la acción deliberada de los estratos más altos (ver MacClure, Barozet y Moya 2015). El mandato del esfuerzo se constituye a través de un reconocimiento de su dificultad: es claro y evidente que el esfuerzo puede fracasar, y que existen barreras importantes, pero se obtiene por ello la conclusión que es posible abandonarlo, lo que se rechaza explícitamente. 
En pocas palabras para surgir uno tiene que esforzarse (murmullos de aceptación) y si te ponen barreras tienes que esforzarte más simplemente hasta que puedas conseguir lo que quieras tener (Joven, NSE Medio-Bajo) (PNUD 2016)

Ahora bien ¿cuán neoliberal resulta este sujeto? Hay múltiples elementos que indicarían que estamos ante la asunción de ese tipo de discurso: Su carácter individual, su sacralización del esfuerzo, su imagen negativa de quienes no se esfuerzan, la imagen que está en el propio sujeto la capacidad de superar todo problema. Como lo mencionan Araujo y Martuccelli: 'En breve, ¿no es esto acaso el corazón del Homo neoliberal, un individuo conminado antes que nada a definirse como un sujeto económico, y por lo tanto como un empresario de sí mismo?' (Araujo y Martuccelli, 2012, II: 57).

Sin embargo, ello no es todo: detrás del mérito no hay univocidad (ha mostrado Dubet, 2009 para el caso francés). Hay una conclusión a la cual la subjetividad se acerca, pero en la cual no se queda: La idea que quien es pobre es porque quiere serlo o que el valor de las personas se mide en sus resultados. En la subjetividad se rechazan esas conclusiones y ahí se establece la diferencia con una lectura neoliberal plenamente 'conversa'. El énfasis de la norma está en el esfuerzo, no en su resultado. La persona se constituye como sujeto en ese enfrentarse a la dificultad -la dificultad es central (así Castillo 2016, por ejemplo, usa 'hazaña' para describir la acción). Más aún, en contraposición con un discurso 'converso', quienes aparecen como exitosos pueden ser vistos como alejados del esfuerzo, y cuando se observa que ellos son los que ponen el límite a otros, se constituye una mirada muy negativa

Como te digo, porque si tu ganancia o tu nivel económico significan menoscabar a los demás, cachai, o haberse estafado a mucha gente, no creo que estés demasiado orgulloso, o defenderías a esas personas cachai y eso es lo que prácticamente hacen los ricos, los ricos de este país (Joven, NSE Medio-Bajo) (PNUD 2016)

En cierto sentido, y expandiendo los conceptos más allá de lo aconsejable, aparece aquí una discusión clásica. El debate sobre la tesis de la Ética Protestante de Weber siempre ha 
discurrido entre quienes interpretan la ética protestante como una ética de la riqueza (Dios premia en la tierra a quienes se esfuerzan), y quienes la interpretan como una ética del esfuerzo (Dios valora el trabajo y el resultado es éticamente indiferente) (Gil Villegas, 2013; Marshall, 1982). La subjetividad que observamos aquí sigue una ética del esfuerzo: es ahí donde se centra el mandato, no en el éxito. No hay valoración necesaria de la riqueza, ni lógica empresarial. La idea del esfuerzo, entonces, no opera como equivalente simple a una subjetivación neoliberal.

Donde más de manifiesto queda la importancia de diferenciar estas formas de identidad del neoliberalismo es en la relación entre mérito y desigualdad. Ya el hecho que es posible inscribirse en una cultura de valoración del esfuerzo y criticar acerbamente a las personas de estratos altos muestra que esta relación puede ser compleja. El discurso del mérito no es un mero justificante de la desigualdad. Existe evidencia que muestra que las personas aceptan altas diferencias de ingreso (Castillo, Miranda, y Carrasco, 2012); y sin embargo al mismo tiempo perciben que la desigualdad existente en Chile es injusta (82\% declara que diferencias de ingreso son demasiado grandes, CEP 2019). La Encuesta CEP mayo 2019 (ver tabla 2) así lo sustenta. A través de dos indicadores, uno sobre igualdad y otro sobre mérito encontramos que un $47 \%$ de la población se ubica al mismo tiempo en un polo pro mérito y en un polo pro-igualdad. El mérito y la igualdad no están en contradicción para buena parte de la población.

Tabla 2. Relación entre mérito e igualdad

\begin{tabular}{ccc}
\hline & + Igualitario & -Igualitario \\
\hline+ Mérito & 47 & 20 \\
\hline -Mérito & 24 & 9
\end{tabular}

Nota. Igualdad: Promedio de (a) distribución injusta y (b) enojo por diferencias ricos y pobres (las escalas de las preguntas se igualaron). De mínimo a punto medio de escala igualitario, más alto que punto medio + igualitario

Mérito: Promedio de (a) responsabilidad de mantenerse es de las personas o del Estado y (b) ingresos más iguales o debe premiarse esfuerzo. De mínimo a punto medio escala mérito, más alto que punto medio + mérito Fuente: Elaboración propia sobre encuesta CEP mayo 2019

Esto fundamenta la diferencia entre evaluación personal y de la sociedad: Si el discurso del esfuerzo es un discurso de superación de las barreras que pone la sociedad entonces todo 
lo alcanzado se debe a la propia persona, y no al contexto país. El juicio experiencial de mejoría en la vida no redunda en una opinión positiva de la sociedad, puesto que el agente de esa mejoría es sólo el esfuerzo personal.

Por lo tanto, la amplia valoración del mérito no implica sustentar el modelo. Si las causas percibidas de la desigualdad no incluyen el mérito (es una desigualdad inmerecida) una cultura de esfuerzo y mérito se transforma en crítica. Incluso esta capa, más cercana al mundo neoliberal, no justifica el orden real del neoliberalismo.

\section{d. Las consecuencias del rechazo moral}

Una cosa es cómo va el mundo, otra cosa es su evaluación. Todas las capas que hemos analizado pueden ser enjuiciadas moralmente y ese juicio es el que abordamos ahora.

Lo que se observa es una crítica al tipo de sociedad que se ha creado en el país. Una sociedad percibida como individualista, materialista, agresiva, consumista (van Bavell y Sell-Trujillo 2007 analizan cómo se usa la idea de "consumismo" para observar y monitorear el consumo en Chile). Se rechaza un mundo hostil, amenazante, donde nunca se puede bajar la guardia, y donde se valora lo que es negativo. Algunos analistas argumentaron que estas críticas son más bien de élite (Tironi, 2000) pero ellas se repiten en estudios cualitativos realizados entre la población general.

Yo creo que industrialmente ha crecido harto en los veinte últimos años Chile, pero culturalmente y educacionalmente ha decaído demasiado (Joven, NSE Medio-Bajo)

En lo material si se puede encontrar de todo, pero en lo humano y emocional se deja todo de lado, en estos momentos lo emocional (NSE Medio) (PNUD Inv. Progreso 2015)

La vida buena y aceptable no es la vida que la sociedad actual promovería. Por el contrario, la vida buena se caracteriza por un vivir humano entre personas (Jiménez 2019) ${ }^{7}$ : En el saludo entre vecinos, en un habitar entre personas que se conocen y re-conocen, en un ritmo

\footnotetext{
${ }^{7}$ El estudio realizó 16 grupos de discusión durante el año 2016 a lo largo del país (Arica a Punta Arenas). Los grupos cubrieron diversos tamaños de ciudad y diferentes bases económicas, manteniendo el segmento analizado: Adultos estrato medio.
} 
de vida para la convivencia y el disfrute. Esa es la vida que la sociedad actual amenaza con una vida agobiante, donde todo es impersonal y vacío de sentido.

pero nosotros aquí no, esa es la gran diferencia que hay, aquí nosotros podemos conversar, nos saludamos, pero allá en la ciudad...ni en XX ya ni se saludan, llevan toda acelerá la vida (Grupo Zona Sur)

Yo vivo, vivía en barrio norte y tengo los mejores recuerdos, todavía nos juntamos con mis amigos de infancia y es como si fueran mis hermanos (Grupo Zona Centro)

Claro es que no, yo soy la señora de, es que trabajo acá y qué se yo. Pero te vas a Santiago y no eres nadie, eres un común, como el resto no más. Y eso duele. (Grupo Zona Norte) (Jiménez 2019)

Mayol et al (2013) analizan esto como parte de un "Chile profundo" en disolución, frente al nuevo del emprendimiento. Sin embargo, en los grupos de discusión el esfuerzo no entra en contradicción con esta visión de la vida deseable ( el esfuerzo no es, recordemos, per se un mandato de emprendimiento).

La sociedad, el modo y forma de vida que se genera en ella, las acciones que nosotros mismo realizamos (las personas saben que se critican a sí mismas), no se valida como algo bueno.

\section{Discusión. Vivir bajo el neoliberalismo}

En síntesis, se observan cuatro capas bien diferenciadas en la relación subjetiva de las personas con el neoliberalismo en Chile en las primeras décadas del siglo XXI. Se diferencian en cuan acordes son con el modelo "neoliberal", sin embargo, incluso cuando se acercan a éste, la dinámica subjetiva marca su diferencia: Siempre que se puede concluir que el modelo es aceptable, las subjetividades tienden a cerrar esa posibilidad. Así en la primera capa se observa un mejor estándar de vida, pero de ahí no se extrae la conclusión que el modelo sea positivo, y así evalúan a la sociedad negativamente. Piensan que el mundo 'opera' neoliberalmente, pero este no sería un mundo de libertad, sino uno de opresión. Piensan que el esfuerzo y el mérito son importantes, y un deber, pero no concluyen que el modelo real se base en el mérito ni ese mandato es uno de emprendimiento capitalista. 
Piensan que la vida neoliberal es una mala forma de vida, que evita que se logre "la vida buena".

Reconocer la complejidad de la subjetividad y evitar ciertas identidades fáciles resulta aquí crucial. No equiparar la legitimidad (¿es bueno el modelo?) con la evaluación y satisfacción directa (¿estoy satisfecho o no?). No inferir creencias a partir de conductas (que las personas compren en centros comerciales es compatible con muchas subjetividades distintas). No aplanar al sujeto es uno de los requisitos de su comprensión.

En primer lugar, es importante evitar reduccionismos. Si las personas valoran la competencia, o aceptan ciertos niveles de desigualdad, no se sigue que acepten en general el modelo: Se puede tener mirada muy positiva del esfuerzo personal y, al mismo tiempo, demandar una mayor participación del Estado en la regulación de la vida social (Castells y Calderón, 2019:37 con datos del Latinobarómetro muestran que los chilenos están entre los más estatistas de América Latina). Más allá, incluso en casos de aceptación 'conversa' del neoliberalismo no se concluye que se acepte su realidad. Personas con una visión neoliberal del esfuerzo (una concepción emprendedora de éste) critican desde ahí la realidad porque la aplicación real no está a la altura de su promesa (ya decía Horkheimer, 2002, que el sujeto crítico es quien toma en serio las promesas y demandas del 'padre', de la 'ley').

En segundo lugar, es necesario recalcar que el plano cognitivo no es el plano moral. Los procesos de naturalización del modelo que ocurrieron en la sociedad chilena no implicaron per se pensar que dicha naturalización fuera positiva. Buena parte de la población se resistió a dar ese salto. No hay necesidad alguna de hacer equivalente lo real y lo bueno.

En tercer lugar, no cabe equiparar resignación con legitimidad. Si no hay alternativa a la realidad actual, entonces no queda más que adaptarse. De lo cual no se sigue que esos comportamientos sean buenos desde el punto de vista de quienes lo realizan. Dado que hay que vivir, se toman las decisiones más prácticas al interior del mundo, pero ellas pueden ser vividas bajo el prisma de la crítica. Al no habitar el mundo deseado, entonces la conducta al interior de él tampoco es necesariamente deseada.

Al principio analizamos la idea de una subjetividad que no se redujera a la legitimidad y esbozamos la importancia de un espacio bajo el cual la subjetividad no sea equivalente a una conducta. 
La subjetividad que hemos analizado requiere ser entendida bajo esos parámetros. Incluye un momento de legitimidad (la dimensión del juicio moral, que es negativo), pero no se reduce a éste. Incluye además un juicio de índole empírica (la constatación de avance), un mapa cognitivo del mundo (la naturalización del modelo) y una forma de pensarse a sí mismo (como un sujeto que se esfuerza y no debe desfallecer en dicho esfuerzo). A su vez, son dimensiones que no se descubren simplemente observando la práctica. Lo que aparece es una subjetividad que no se entiende fuera de un mundo neoliberal, y que al mismo tiempo no es un plegamiento de la subjetividad a dicho mundo.

Las lecturas directas esconden todas las dificultades que experimenta la subjetividad: Que el mandato del esfuerzo no es un mandato de éxito ni equivale a una ética empresarial de crecimiento. $\mathrm{O}$ el hecho que el mandato del esfuerzo, la idea que todo se debe a uno mismo, desarticula cualquier relación entre la constatación de un aumento en el estándar de vida y el juicio sobre la sociedad. También dificulta percibir que el mundo naturalizado no es leído en forma 'canónica': No observan las personas un mundo de sujetos libres sino uno de sujetos coaccionados a seguir una máquina (una 'ley de la selva'). La lectura directa dificulta así dar cuenta de las variantes y vericuetos que existen tras juicios y haceres.

Con ello volvemos a un tema básico: Si esta es la visión que las personas han mantenido sobre el neoliberalismo y éste ha sido el modelo operativo cerca de cuarenta años, ¿cómo fue posible la reproducción? La visión teórica tradicional, como vimos, al respecto es directa: La subjetividad reproduce el sistema cuando se alinea positivamente hacia este, no lo hace en caso contrario. Si no fuera así la subjetividad no sería relevante y las dinámicas sociales operarían fuera de los procesos subjetivos.

Lo que argüiremos es que las dinámicas subjetivas son relevantes para la reproducción social en Chile, sólo que no lo hacen por aceptación de la estructura. No es que la reproducción del modelo opere a pesar de una subjetividad que la rechaza, sino que ocurre a través de esa subjetividad refractaria. Afirmamos que la forma en que la población chilena ha intentado responder a un mundo hostil y negativo es a través de su escape, buscando un refugio (algo puntualizado desde temprano, así Lechner en Los patios interiores de la democracia de 1987, 2007, I: 339-470).

La comparación con el cristianismo primitivo realizada con anterioridad tiene su fundamento aquí: También aparece ahí un mundo al cual el sujeto se adapta, dentro del cual 
se realizan sus lógicas, pero se intenta que estas acciones no afecten la propia identidad. La persona que uno es se construye en otro lugar que el mundo. En eso consistía la diferencia agustiniana entre la ciudad de los hombres y la ciudad de Dios; y si bien se actuaba en la primera, era la segunda la que orientaba la subjetividad (y al largo plazo, la acción).

En el caso chileno, el escape consistió en retraerse a la esfera doméstica y familiar, convertidos en lugar subjetivo central (Araujo y Martuccelli, 2012; Tironi, 2005). Es importante observar que la familia se construyó como un espacio emocional, no como estructura o patrón de autoridad: Familia es el lugar donde se es querido y apoyado ${ }^{9}$. Esto hace que la centralidad de la familia no tenga necesariamente un talante conservador, de defensa y conservación de la familia tradicional (la familia tradicional se crea sobre la base de una autoridad, no de una emoción; no del amor sino de la ley), y que sea compatible con un debilitamiento de su forma tradicional. La familia se define desde emociones (cariño, amor, acogida); y sólo así tiene sentido la retirada a ella, sólo así puede operar como refugio frente al mundo.

Esta retirada produce la conexión con la reproducción: Esa inmersión en la vida familiar genera conductas que reproducen el modelo. El refugio debe ser construido, y construir un refugio frente a los males del mundo implica construir un buen refugio. Esa vida deseada requiere una serie de prácticas (reuniones familiares por ejemplo), una materialidad del hogar (remodelar el hogar es una actividad común), al fin consumo. Más aún, la relación culposa con el consumo desaparece dentro de la familia, en particular con los hijos: hacia ellos adquiere sentido no tener límites (Castillo 2016: 224-5). Las relaciones con las personas, el centro de la vida buena en la visión de la población, requieren una base material y rituales de consumo.

Obtener esos niveles de consumo implica a su vez importantes niveles de esfuerzo laboral: el trabajo agobiante es la única forma de poder construir el hogar deseado. Las personas no sienten que el trabajo sea un fin, y el tiempo de trabajo es un tiempo desvalorizado en relación con el familiar; pero ese tiempo permite que la familia tenga lo que ella merece. La

\footnotetext{
${ }^{99}$ En una entrevista que el autor realizó sobre pensiones, una de las personas mencionaba que pensaba resolver su jubilación con la idea que la apoyarían las 'personas que estaban obligadas a quererla'. Esa casi aporía de la obligación de querer es la imagen fundamental de la vida familiar en Chile.
} 
alta valoración de la familia justifica los altos niveles de esfuerzo laboral (Araujo y Martuccelli, 2012).

Las consecuencias del retiro al espacio familiar-doméstico, que provienen del rechazo del mundo, son -entonces- una conducta de trabajo y de consumo que son exactamente las que el modelo requiere de las personas. No ayudan a reproducirlo en la manera de una válvula de escape (contra Wallerstein 2004: 37), sino que son parte del proceso que genera las acciones requeridas. El rechazo al mundo neoliberal, la falta de plegamiento del sujeto, a través de las consecuencia práctica de la búsqueda del refugio familiar, produce la reproducción del modelo rechazado. No hay relación directa entre legitimidad y reproducción y al mismo tiempo la subjetividad es relevante para la operación y continuidad de la estructura. El neoliberalismo construyó su reproducción a través de su falta de legitimidad.

\section{Conclusión.}

Tenemos entonces una subjetividad formada bajo el neoliberalismo, con elementos cercanos a éste, que no es una subjetividad adherida a él. Existe una distancia en cada una de las capas mencionadas a una subjetividad neoliberal pura. Y en esa distancia se produce la reproducción del sistema. La subjetividad no es ni apoyo directo al modelo ni es irrelevante para su operación, sino que se hace parte de la dinámica de reproducción social con la diferencia entre subjetividad y modelo.

Frente al neoliberalismo los chilenos nunca fueron panglossianos, que creían que vivían en el mejor de los mundos posibles, como el carácter del Cándido de Voltaire. Al igual que el protagonista homónimo de la novela citada, frente a los problemas del mundo concluyeron que lo mejor era retirarse a cultivar su jardín. Pero este retiro, que no cambiaba activamente el mundo y que lo reproducía, nunca fue aceptación.

Las recientes protestas de 2019/2020 muestran los límites de ese proceso de estabilización. El mecanismo de reproducción social expuesto aquí reveló su fragilidad en esos momentos críticos: cuando fue patente para todos que la insatisfacción con el modelo era amplia, y que la acción colectiva era posible. El Cándido de Voltaire se publicó en 1759, y treinta años más tarde aquellos a quienes se recomendó el cuidado del jardín realizaron una revolución. 
Ahora, si el error previo fue leer en cada consumidor de centro comercial alguien que apoyaba al modelo, cabe ahora tener los resguardos análogos y hacernos la misma pregunta en relación con estas protestas. Si no era cierto que ir al mall era aceptar el modelo, ¿podemos concluir que quien protesta sólo se relaciona con el modelo para transformarlo? La complejidad de la subjetivación debe aplicarse en ambas direcciones.

Lo que se desarrolló en Chile fue una forma de 'astucia del neoliberalismo': Un modelo que durante décadas no solo convivió con su falta de aprobación, sino que logró reproducirse usándolo. Sus detractores no debieran olvidar esas capacidades de cooptación de la crítica. Esta flexibilidad aplica en general al capitalismo (Welsh 2019: 4-5), y bien podemos recordar cómo éste ha sobrevivido, una y otra vez, a los agoreros de su inminente final.

\section{Referencias}

Acuña, E., y Pérez, E. (2005). Trayectorias Laborales. El tránsito entre el trabajo asalariado y el trabajo independiente (Cuadernos de Investigación 23). Santiago: Dirección del Trabajo.

Agamben, G. (2016). Homo Sacer. L’Integrale 1997-2015. París: Éditions du Seuil.

Appel, H., \& Orenstein, M. A. (2016). Why did Neoliberalism Triumph and Endure in the Post-Communist World? Comparative Politics, 48(3), 313-331.

Araujo, K. (2014). 'La desmesura y sus sujetos: el trabajo en el caso de Chile' en A. Streher y L. Godoy (Eds.), Transformaciones del Trabajo, Subjetividad e Identidades (p. 277298). Santiago: RIL.

Araujo, K., y Martuccelli, D. (2012). Desafios comunes. Santiago: LOM.

Aravena, A., y Baeza, M. A. (2015) Construcción socio-imaginaria de relaciones sociales: la desconfianza y el descontento en el Chile post-dictadura. Cinta moebio (53): 147-157

Atkinson, W. (2010). Phenomenological Additions to the Bourdieusian Toolbox. Sociological Theory, 28(1), 1-19.

Barozet, E., y Fierro, J. (2014) La clase media en Chile. Revista Paraguaya de Sociología, $51(145), 147-158$

Beyer, H. (2015). Diagnósticos alternativos sobre la crisis de confianza. Estudios Públicos 40, 107-136. 
Bockman, J. (2019). Democratic Socialism in Chile and Peru: Revisiting the "Chicago Boys" as the Origin of Neoliberalism. Comparative Studies in Society and History, 61(3), 654 $-679$.

Bolt, J.; and Inklaar, R.; and de Jong, H.; and van Zanden, J. L. (2018) Maddison Project. Database, version 2018, Maddison Project Working paper 10.

Boltanski, L. (200) De la critique. Paris: Gallimard

Bourdieu, P. (1980) Le sens pratique. Paris: Minuit

Bourdieu, P. (2000). Las Estructuras Sociales de la Economía. Buenos Aires: Manantial.

Bourdieu, P. (2015) Sociologie générale. Volume I. París: Raisons d'agir/Seuil

Bröckling, U. (2015). El self emprendedor. Santiago: Ediciones Universidad Alberto Hurtado.

Brunner, J. J. (1981). La Cultura Autoritaria. Santiago: FLACSO.

Brunner, J. J. (1983) Entrevistas, discursos, identidades Santiago: FLACSO

Calderón, F. y Castells, M. (2019) La nueva América Latina. Santiago: Fondo de Cultura Económica.

Canales, M. (2019). Neoliberalismo subjetivo en la sociedad chilena. Manuscrito sin publicar, Departamento de Sociología. U. de Chile

Castells, M. (2005) Globalización, desarrollo y democracia. Chile en el contexto mundial. Santiago: Fondo de Cultura Económica.

Castillo, J. C., Miranda, D., y Carrasco, D. (2012). Percepción de Desigualdad Económica en Chile. Psykhe (Santiago), 21 (1), 99-114.

Castillo, M. (2016) Fronteras simbólicas y clases medias. Movilidad social en Chile. Perfiles Latinoamericanos, 24(8), 213-241

CEP. (2016). ¿Malestar en Chile? Informe Encuesta CEP 2016. Santiago: Centro de Estudios Públicos.

CEP (2019) Encuesta mayo 2019. Santiago: Centro de Estudios Públicos. Disponible en https://www.cepchile.cl/cep/encuestas-cep/encuestas-2009-2018/estudio-nacional-deopinion-publica-mayo-2019

Clark, T. (2017). Rethinking Chile's “Chicago Boys”. Third World Quarterly, 38(6), 13501365 . 
Clarke, S. (2005). 'The neoliberal theory of society' en A. Saad-Filho y D. Johnston (Eds.), Neoliberalism: A critical reader (p. 50-59). Londres: Pluto Press.

Deleuze, G. (1991). Qu 'est-ce que la philosophie? Paris: Editions de Minuit.

Dubet, F. (1994) Sociologie de l'expériencie. Paris: Éditions du Seuil.

Dubet, F. (2009). Injustice at Work Boulder, CO: Paradigm Publishers.

Dunn, B. (2016) Against neoliberalism as a concept. Capital \& Class, 41 (3): 435-454

Durand, J-P. (2004) La chaîne invisible. Paris: Éditions du Seuil

Elster, J. (1989) The cement of society. Cambridge: Cambridge University Press

Fagioli, A. (2018) Acumulación originaria y capitalismo neoliberal. Una posible lectura del Chile post-golpe. Isegoria, 59: 573-593

Falabella, A. (2019) The ethics of competition: accountability policy enactment in Chilean school's everyday life. Journal of Educational Policy DOI: 10.1080/02680939.2019.1635272

Foucault, M. (2000). Defender la Sociedad. Buenos Aires: Fondo de Cultura Económica.

Foucault, M. (2007). Nacimiento de la Biopolítica. Buenos Aires: Fondo de Cultura Económica.

Gaete, T. y Soto, A. (2012) Esta es mi trayectoria, este es mi trabajo: Narrativas e Identidad en el trabajo en Chile. Psykhe, 21 (2): 47-59

Gárate Chateau, M. (2012). La Revolución Capitalista en Chile (1973-2003). Santiago: Ediciones Universidad Alberto Hurtado

Garretón, M. A. (2012). Neoliberalismo corregido y progresismo limitado: los gobiernos de la Concertación en Chile. 1990-2010. Santiago: Arcis / CLACSO.

Gil Villegas, F. (2013). Max Weber y la guerra académica de los 100 años. Ciudad de México: Fondo de Cultura Económica y Colegio de México.

Gray, J. (1998) False Dawn. The delusions of global capitalism. London: Granta Books Habermas, J. (1998). Facticidad y Validez. Madrid: Trotta.

Horkheimer, M. (2002). Crítica de la Razón Instrumental. Madrid: Trotta

Hosbawm, E. (1975). The Age of Capital, 1848-1875. Londres: Nicolson Ltd.

Jiménez, J. (2016) Tres investigaciones sobre la pertinencia intelectual de la sociología. Tesis de Doctorado en Ciencias Sociales, Universidad de Chile. 
Jiménez, J. (2019) Los significados del territorio en Chile. ¿Qué se dice cuando se habla del lugar en el cual se vive? Ponencia para XII Congreso ALAS Lima. Diciembre 2019

Jorrat, J. (2014) Percepción de clase y percepción de desigualdad en la Argentina en un contexto internacional con especial referencia a las clases medias, pp 55-85 en Adamovsky, E.; Visacovsky, S.; y Vargas, P. (comps.) Clases Medias: Nuevos Enfoques desde la sociología, la historia y la antropología. Buenos Aires: Ariel

King, A. (2000). Thinking with Bourdieu against Bourdieu: A 'Practical' Critique of the Habitus. Sociological Theory, 18(3), 417-433.

Laval, C., y Dardot, P. (2013). La Nueva Razón del Mundo Barcelona: Gedisa.

Lechner, N. (2007). Obras escogidas. Santiago: LOM.

Lichbach, M. y Seligman, A. (2000) Market and Community. University Park, PA: The Pennsylvania State University Press.

Lordon, F (2013) La Société des affects. Paris: Seuil.

MacClure, O; Barozet, E.; y Moya, C. (2015) Juicios de las clases medias sobre la élite económica: Polis, 14 (41), 435-460

MacClure, O; Barozet, E.; Ayala, M. C.; Moya, C.; Valenzuela, A. M. (2019) Encontrar la posición de uno mismo en la sociedad. Revista Brasileira de Ciências Sociais, 34 (99), $1-28$

Márquez, R. (2015). La distancia entre la ciudadanía y las élites: una mirada desde el informe Desarrollo humano en Chile. Estudios Públicos, 40, 83-106.

Marshall, G. (1982). In search of the spirit of Capitalism Londres: Hutchinson.

Martínez, J. and Palacios, M. (1996). Informe sobre la decencia. Santiago: SUR

Martuccelli, D. (2013). Sociologías de la Modernidad. Santiago: LOM

Mayol, A. (2012). El derrumbe del Modelo. Santiago: LOM.

Mayol, A.; Azócar O., C.; Azócar R., C. (2013) El Chile profundo. Santiago: Liberalia

Medina-Zárate, J. y Uchôa de Oliveira, F. (2019) Why should we be interested in the specificity of subjectivity and neoliberalism in Latin America? Subjectivity, 12: 281-287

Milanovic, B. (2019) Capitalism Alone. Cambridge, Mass: Harvard University Press.

Mokyr, J. (2009) The Enlightened Economy: An Economic History of Britain 1700-1850. London: Penguin 
Morandé, P. (2017) Cultura y Modernización en América Latina. Santiago: Instituto de Estudios de la Sociedad. Original de 1984.

Moulian, T. (1997). Chile actual: Anatomía de un Mito. Santiago: LOM.

Navia, P. (1998) 'Creando poder popular: la inclusión y el pluralismo político en Chile' en Figueroa, M.; and Vicuña, M. (comps.) El Chile del Bicentenario (p 275-309). Santiago: Ediciones Universidad Diego Portales

Oppliger, M., y Guzmán, E. (2012). El Malestar en Chile. ¿Teoría o Diagnóstico? Santiago: RIL.

Osorio, J. (2004) El estado en el centro de la mundialización. Ciudad de México: Fondo de Cultura Económica

PNUD. (1998). Las Paradojas de la Modernización. Santiago: PNUD.

PNUD (2000) Más sociedad para gobernar el futuro. Santiago: PNUD

PNUD. (2002). Nosotros los chilenos. Santiago: PNUD.

PNUD. (2004). El poder, ¿para qué y para quién? Santiago: PNUD.

PNUD. (2015). Los Tiempos de la Politización. Santiago: PNUD.

PNUD. (2016). Del mandato del esfuerzo en los discursos grupales en el Chile del 2015. En IX Congreso Chileno de Sociología. Talca.

PUC (2018) Encuesta Bicentenario 2018. Santiago: Pontificia Universidad Católica

Reygadas, L. (2015) 'Más allá de la Legitimación' en Castillo, M. and Maldonado, C. Desigualdades (p 39-68). Santiago: RIL

Roper, J., Ganesh, S., y Inkson, K. (2011). Neoliberalism and knowledge interests in boundaryless careers discourse. Work. Employment \& Society, 24 (4), 661-679.

Salazar, G. (2000). Labradores, peones y proletarios. Santiago: LOM.

Salazar, G. (2009). Mercaderes, empresarios y capitalistas. Santiago: Sudamericana.

Tironi, E. (2000). La irrupción de las masas y el malestar de las élites. Santiago: Grijalbo

Tironi, E. (2005). El sueño chileno Santiago: Taurus.

Touraine, A. (2013). La Fin des Sociétés. Paris: Seuil

Undurraga, V. (2012). Los Rostros del Honor. Santiago: Centro de Investigaciones Barros Arana

Van Bavell, R., y Sell-Trujillo. L. (2007) Understandings of Consumerism in Chile. Journal of Consumer Culture, 3 (3), 343-362 
Venugopal, R. (2015) Neoliberalism as a concept. Economy and Society, 44 (2): 165-187

Wagner, P. (1997). Sociología de la Modernidad Barcelona: Herder.

Welsh, J. (2019). Struggling beyond the paradigm of Neoliberalism. Thesis Eleven, DOI 072551361988866. doi:10.1177/0725513619888667

White, H. C. (2008). Identity and Control. Princeton, NJ: Princeton University Press.

Žižek, S. (2008) El espectro de la ideología ¿Critica de la ideología hoy? pp 7-42 en Žižek, S. (Comp.). Ideología. Un mapa de la cuestión. Buenos Aires: Fondo de Cultura Económica 